\section{P253 INVESTIGATING THE COMPLEXITY OF THE RELATIONSHIP BETWEEN GASTRO-OESOPHAGEAL REFLUX AND CF LUNG DISEASE}

${ }^{1} \mathrm{RW}$ Lord, ${ }^{2} \mathrm{~S}$ Treadway, ${ }^{2} \mathrm{~S}$ Pearson, ${ }^{1} \mathrm{PJ}$ Barry, ${ }^{1} \mathrm{~B}$ Bianco, ${ }^{2} \mathrm{PJ}$ Whorwell, ${ }^{2} \mathrm{RB}$ Jones, ${ }^{3} \mathrm{PS}$ McNamara, ${ }^{4} \mathrm{R}$ Beynon, ${ }^{2} \mathrm{JA}$ Smith, ${ }^{1} \mathrm{AM}$ Jones. ${ }^{1}$ Manchester Adult Cystic Fibrosis Centre, Manchester, UK; ${ }^{2}$ University Hospital South Manchester, Manchester, UK; ${ }^{3}$ Alder Hey Childrens Hospital, Manchester, UK; ${ }^{4}$ University of Liverpool, Liverpool, UK

\subsection{6/thoraxjnl-2017-210983.395}

Background There has been a suggested connexion between gastro-oesophageal reflux (GOR) and CF lung disease. Lung disease can result in increasingly negative inspiratory thoracic pressures. These create gastro-oesophageal pressure gradients along which gastric contents may move. Then if aspirated reflux may have the potential to cause lung damage. We aimed to assess if there is a relationship between GOR and markers of lung disease severity.

Methods We are conducting a prospective observational study in stable adult CF patients, measuring GOR with combined $\mathrm{pH}$ and impedance (pH-MII). In preliminary analyses, we have compared reflux measures (total, proximal, supine and supine proximal events) and retrospective data including routinely collected lung function and number of courses of intravenous antibiotics.

Results 51 patients were recruited with 36 patients (mean age 30 years, mean FEV1 54\% predicted, 28 males) completing all measures. Total number of reflux episodes were increased in 58\% (median 81, IQR 55-105) compared to established normative values ( $<75$ episodes). 'High risk' reflux (increased proximal or supine proximal events) was noted in $47 \%$. Increasing reflux and higher number of IV courses in the preceding year displayed a positive trend $(r=0.300, p=0.075)$, but this relationship was not seen for number of courses over 2 years. Curiously, patients with greater numbers of reflux events exhibited less decline in lung function over the preceding year $(\mathrm{r}=0.416, \mathrm{p}=0.016)$. None of the reflux measures were correlated with baseline lung function.

Conclusions We have demonstrated that patients with stable CF lung disease have high rates of total reflux events in comparison to normative values. Almost half have reflux which has the potential to be high risk for aspiration. There was a suggestion that increased reflux events maybe associated with increased exacerbation risk. However our data also suggests, in complete contrast, that increased reflux relates to less progression of lung disease - this is not in keeping with commonly held beliefs. Further studies will be needed to unpick what is undeniably a complex relationship.

\section{P254 FEASIBILITY OF ULTRASHORT ECHO TIME (UTE) MRI TO EVALUATE THE EFFECT OF LUMACAFTOR/IVACAFTOR THERAPY IN CHILDREN WITH CYSTIC FIBROSIS (CF) HOMOZYGOUS FOR F508DEL}

${ }^{1} S$ Nagle, ${ }^{2} \mathrm{AS}$ Brody, ${ }^{2} \mathrm{~J}$ Woods, ${ }^{1} \mathrm{KM}$ Johnson, ${ }^{3} \mathrm{~L}$ Wang, ${ }^{3} \mathrm{G}$ Marigowda, ${ }^{3} \mathrm{D}$ Waltz, ${ }^{4} \mathrm{~J}$ Goldin, ${ }^{5} \mathrm{~F}$ Ratjen, ${ }^{3} \mathrm{C}$ Hug. ${ }^{1}$ University of Wisconsin, Madison, US; ${ }^{2}$ Cincinnati Children's Hospital Medical Centre, Cincinnati, US; ${ }^{3}$ Vertex Pharmaceuticals Incorporated, Boston, US; ${ }^{4}$ David Geffen School of Medicine, Los Angeles, US; ${ }^{5}$ University of Toronto, Toronto, Canada
Objective To evaluate ultrashort echo time (UTE) magnetic resonance imaging (MRI) as a feasible endpoint in a multicenter trial using a subset of patients aged 6 to 11 years with cystic fibrosis (CF) homozygous for the F508del mutation treated with lumacaftor/ivacaftor (LUM/IVA) combination therapy (NCT02514473).

Methods UTE MRI scans were obtained at study baseline $(n=10)$; a second scan was completed at week 24 in 9 subjects (4 active treatment, 5 placebo) at 3 institutions using MRI hardware from 2 vendors. One of the platforms was 3D radial UTE (Johnson et al. Magn Reson Med. 2013;70:12411250); the other was 3D "stack of stars" UTE (Lederlin et al. J Magn Reson Imaging. 2014;40:839-847). Scans were obtained during $5 \mathrm{~min}$ of tidal breathing without the use of intravenous contrast. MRI scans were scored by 2 independent readers using the Brody score after supervised training on UTE MRI scans. Readers were blinded to time point and treatment group. Scores are presented as mean (SD); no statistical testing was performed.

Results Despite the lower image quality of MRI compared with computed tomography, potential treatment effects were seen on the UTE MRI images (figure 1A). Mean total Brody score decreased from 41.1 to 32.5 with treatment, a change from baseline (SD) of 8.4 (11.2) with active treatment, but increased from 31.3 to 34.6 , a change from baseline of 3.3 (8.2) with placebo (figure 1B). Mucus plugging subscore decreased by 5.0 (5.1) from 8.5 to 3.5 with active treatment but increased by 1.4 (4.4) from 4.2 to 5.6 with placebo. There were no noticeable changes in other subscores (bronchiectasis, peribronchial thickening, parenchyma, or hyperinflation).

Conclusion In this analysis from an exploratory substudy in patients with CF aged 6 to 11 years homozygous for F508del, UTE MRI was a feasible approach for detecting the effect of LUM/IVA, despite the small sample size, short duration of treatment, and limitations in image quality. As optimisation in UTE MRI technology improves image quality, monitoring disease course in patients with CF may improve.

Please refer to page A259 for declarations of interest in relation to abstract P254.

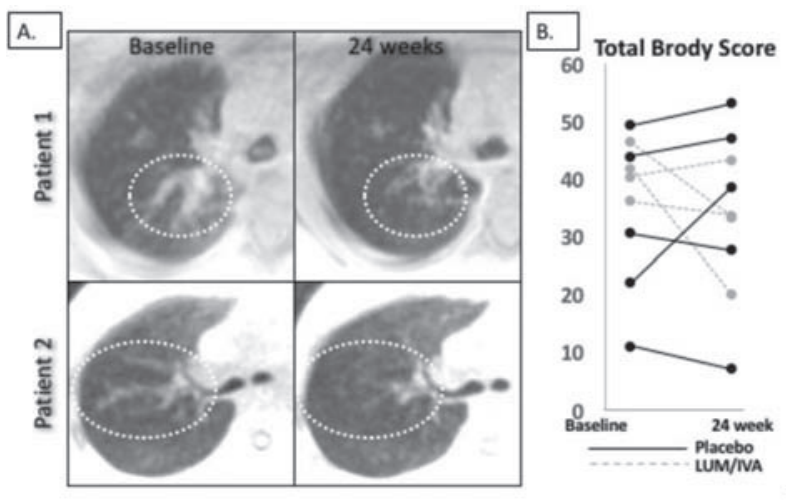

Abstract P254 Figure 1 